\title{
Propriedades microestruturais de argamassas de cimento Portland com adições minerais e poliméricas utilizadas na fixação de porcelanato
}

\section{(Study of the influence of additions on the adherence between polymer- modified mortars and porcelain stoneware tiles)}

\author{
A. E. F. S. Almeida ${ }^{1}$, E. P. Sichieri ${ }^{2}$ \\ ${ }^{1}$ Interunidades em Ciência e Engenharia dos Materiais, USP \\ Av. Dr. Carlos Botelho, 2220, apto. 51, S. Carlos, SP 13560-250 \\ ${ }^{2}$ Departamento de Arquitetura e Urbanismo, EESC, USP \\ Av. Trabalhador S. Carlense, 400, S. Carlos, SP 13566-590. \\ aefsouza@ig.com.br
}

\begin{abstract}
Resumo
Apesar de suas excelentes características técnicas e estéticas, a aplicação do porcelanato requer especial atenção quanto aos procedimentos de execução, devido ser um material diferente das cerâmicas convencionais, principalmente quando aplicados às fachadas as quais estão sujeitas a diversos agentes capazes de danificar os revestimentos cerâmicos. Podem ser encontrados na literatura trabalhos científicos que estudam as propriedades das argamassas colantes para assentamento de materiais cerâmicos com absorção de água superior a 3\%, o que não é observado para os porcelanatos que apresentam absorção de água próxima de zero. Essa é a principal causa da perda ou falta de aderência entre o tardoz da placa de porcelanato e a argamassa, pois não permite o mecanismo de aderência mecânica existente nas cerâmicas porosas. O presente trabalho apresenta algumas propriedades de argamassas com adições de polímero e sílica ativa para a fixação de porcelanato, contribuindo para solucionar os problemas encontrados quanto a falta de aderência. A adição combinada de polímero e sílica ativa às argamassas resulta em excelentes propriedades, ideais para reparos e revestimentos que exigem elevado desempenho, o que viabiliza seu estudo visando a fixação de porcelanato. Os procedimentos experimentais para a determinação da aderência seguiram as prescrições constantes na NBR 14084 - Argamassa colante industrializada para assentamento de cerâmica - Determinação da resistência de aderência. Os resultados obtidos evidenciaram a importância da continuidade do estudo, além da aplicabilidade das argamassas especiais quanto a fixação do revestimento cerâmico em estudo. São apresentadas micrografias das amostras fraturadas das argamassas obtidas por microscopia eletrônica de varredura, assim como o diâmetro médio dos poros das argamassas obtido por porosimetria por intrusão de mercúrio.
\end{abstract}

Palavras-chave: porcelanato, argamassa, aderência, polímero, sílica ativa.

\begin{abstract}
Despite the excellent characteristics of porcelain tiles, its application on building façades requires special attention, since this material differs from conventional ceramics and because façades are exposed to weathering that can damage ceramic revetments. Literature presents lots of researches about adhesive mortars properties employing ceramic tiles with higher water absorption (more than $3 \%$ ). In a different way, we find about porcelain stoneware tiles with almost $0 \%$ water absorption, that is the most important cause of detachments between the back side of porcelain tiles and the mortar, for the reason that there is no mechanical adherence how occurs for porous ceramic tiles. The present study shows some properties of mortars with polymer and silica fume additions to fix porcelain tile, intending to solve the problems about the lack of adherence. The combination of polymer and silica fume to mortars results in excellent properties, which are ideal for repairs and revestments requiring high performance, thus justifying its study for the fixing of porcelain tiles. Procedures were carried out following the specifications of the NBR 14082 standard. This article presents adherence values for mortars containing different amounts of added polymer and silica, indicating the applicability of these mortars as construction material. Microstructural properties were based on mercury intrusion porosimetry tests and scanning electron microscopy of fractured samples.
\end{abstract}

Keywords: porcelain tile; mortar, microstructure, porosity.

\section{INTRODUÇÃO}

Oprodutoporcelanatoapresenta excelentes características técnicas e estéticas resultantes de um processo altamente tecnológico para a sua produção. O porcelanato nacional é o resultado do processamento de matérias-primas de alta pureza, submetidas a um tratamento térmico de $1250{ }^{\circ} \mathrm{C}$ e pressões de compactação acima das usadas nos materiais 
Tabela I - Características técnicas do porcelanato: confronto entre valores requeridos pelas normas e os reais dos produtos comerciais [1].

[Table I - Technical characteristics of porcelain stoneware tile: comparative analyze between standard values and commercial products [1].]

\begin{tabular}{|c|c|c|c|}
\hline Características & Normas & Valores Prescritos & Valores reais/produtos \\
\hline Absorção de água & EN 99 & $\leq 0,5 \%$ & $\leq 0,1 \%$ \\
\hline Resistência à flexão & EN 100 & $\geq 27 \mathrm{~N} / \mathrm{mm}_{3}^{2}$ & $>\overline{50 \mathrm{~N} / \mathrm{mm}_{3}^{2}}$ \\
\hline Resistência à abrasão & EN 102 & $<205 \mathrm{~mm}^{3}$ & $<130 \mathrm{~mm}^{3}$ \\
\hline Resistência ao congelamento & EN 202 & Sem defeitos visíveis & Sem defeitos visíveis \\
\hline Expansão térmica linear & EN 103 & $\leq 9 \times 10^{-6}{ }^{\circ} \mathrm{C}^{-1}$ & $\approx 7 \times 10^{-6}{ }^{\circ} \mathrm{C}^{-1}$ \\
\hline Resistência ao ataque químico & EN 106 & Nenhuma variação visível & Nenhuma variação visível \\
\hline Choque térmico & EN 104 & Nenhuma alteração & Nenhuma alteração \\
\hline Dureza Mohs & EN 101 & $>5$ & $7-8$ \\
\hline Resistência a manchas & EN 122 & Nenhuma variação visível & Nenhuma variação visível \\
\hline
\end{tabular}

cerâmicos convencionais [1]. A elevada compactação resulta um produto com baixa absorção de água, denso, homogêneo, com valores superiores de resistência à abrasão, resistência à ação de ácido, álcalis, gelo e alta resistência à ruptura. As características técnicas dos porcelanatos podem ser observadas na Tabela I [2].

As argamassas para assentamento de materiais cerâmicos são conhecidas como argamassas colantes ou argamassas adesivas, caracterizadas pela aplicação em camada fina e formação de cordões. Estas argamassas são industrializadas e fornecidas na forma de mistura pré-dosada em pó, sendo que a composição varia conforme o fabricante e tipo de argamassa.

Sendo um material em fase inicial de expansão para aplicação na construção civil, principalmente no Brasil, o porcelanato requer procedimentos específicos para sua aplicação, tal como a especificação correta da argamassa de assentamento. As propriedades das argamassas podem ser incrementadas pela adequada incorporação de aditivos ou adições. As propriedades reológicas e mecânicas dos concretos e argamassas variam de acordo com os materiais constituintes, procedimento de execução e condições ambientais no momento do preparo, além da cura.

As argamassas modificadas com polímero têm sido objeto de estudo em diversas pesquisas no Brasil e em outros países, constatando-se que estas adições podem melhorar significativamente as propriedades de compósitos de cimento Portland, entre os quais destacam-se as argamassas. Os polímeros são adicionados na forma de dispersão (látex ou emulsão) às argamassas de cimento Portland durante a mistura. O látex consiste na dispersão de partículas esféricas de polímero orgânico em água, formando um fluído leitoso, geralmente branco, sendo as seguintes propriedades as mais importantes para a adequada aplicação: teor de sólidos, $\mathrm{pH}$, viscosidade, tipo de estabilizador e temperatura mínima de formação de filme [3, 4].

Argamassas com adições de polímero apresentam boas propriedades no estado fresco, tais como aumento na incorporação de ar resultando na melhora da trabalhabilidade e capacidade de retenção de água, favorecidas pela formação de filme ou membrana polimérica que restringe a perda de água por evaporação para o ambiente e por absorção pelo substrato, aumentando o tempo de hidratação do cimento [5].

A resistência de aderência é influenciada significativamente pela proporção de polímero/cimento, pela condição de cura e pelas propriedades do substrato. A modificação dos concretos e argamassas é resultante da hidratação do cimento Portland e da coalescência das partículas de polímero formando filmes contínuos ou membranas que se ligam aos géis hidratados do cimento e aos agregados, constituindo uma cadeia monolítica na qual a fase polímero interpenetra os compostos hidratados do cimento Portland. Esta fase polimérica é a responsável pela diminuição da permeabilidade e pelo aumento da aderência entre a pasta e o agregado [6,7].

A incorporação de sílica ativa às argamassas e concretos resulta em duas formas fundamentais de atuação [8].

- Atuação física: caracterizada pelo denominado efeito filler. As partículas de sílica ativa preenchem a curva granulométrica na faixa inferior a granulometria do cimento, pois consistem em partículas cujo diâmetro médio é aproximadamente cem vezes inferior que o diâmetro das partículas de cimento. Este mecanismo reduz os vazios existentes, resultando no aumento de coesão e compacidade da argamassa ou da fase pasta-agregado, quando em concreto;

- Atuação química: pela reação pozolânica. A sílica ativa consiste num material altamente pozolânico, isto é, de alta reatividade, capaz de reagir com o hidróxido de cálcio $\left(\mathrm{Ca}(\mathrm{OH})_{2}\right)$ para a formação de silicato de cálcio hidratado (C-S-H), responsável pela resistência da pasta de cimento hidratado.

Dentre suas características, o teor muito alto de $\mathrm{SiO}_{2}$, o estado amorfo e a extrema finura da sílica ativa são 
as responsáveis pela sua alta reatividade. No Brasil são encontradas diversas pesquisas sobre as propriedades de argamassas com adição de polímeros. Entretanto, são poucos os trabalhos que abordam especificamente argamassas para porcelanatos. Em estudos recentes foi relatado que a adição conjunta de sílica ativa e polímero acrílico proporciona argamassas com excelentes propriedades, ideais para utilização em reparos e revestimentos que exigem elevado desempenho $[9,10]$.

\section{MATERIAIS E MÉTODOS}

\section{Caracterização dos materiais}

Os materiais foram caracterizados segundo normas nacionais e internacionais, sendo que algumas informações foram fornecidas pelos fabricantes, conforme descritos a seguir.

\section{Aglomerantes}

Para a confecção do substrato padrão foi utilizado o CP II E 32 e para a produção da argamassa colante foi utilizado o CP V ARI Plus. As características químicas e físicas destes materiais são observadas nas Tabelas II e III, conforme dados fornecidos pelo fabricante.

A área da superfície específica da amostra da sílica ativa empregada resultou em $27,74 \mathrm{~m}^{2} / \mathrm{g}$, obtido por ensaio BET.

\section{Agregados}

Para o preparo das argamassas foi empregada a areia fina com diâmetro máximo característico de $0,6 \mathrm{~mm}$, classificado de acordo com a NBR 7217 como areia muito, e módulo de finura de 1,37.

Tabela II - Análise química dos cimentos.

[Table II - Chemical analysis of cement.]

\begin{tabular}{ccc}
\hline $\begin{array}{c}\text { Composição } \\
\text { química }\end{array}$ & $\begin{array}{c}\text { CPV-ARI-Plus } \\
\%\end{array}$ & $\begin{array}{c}\text { CPII-E-32 } \\
\%\end{array}$ \\
\hline Perda ao fogo & 3,10 & 5,08 \\
$\mathrm{SiO}_{2}$ & 18,99 & 23,49 \\
$\mathrm{Al}_{2} \mathrm{O}_{3}$ & 4,32 & 7,22 \\
$\mathrm{Fe}_{2} \mathrm{O}_{3}$ & 3,00 & 2,48 \\
$\mathrm{CaO}$ total & 64,75 & 55,85 \\
$\mathrm{MgO}$ & 0,68 & 2,76 \\
$\mathrm{SO}_{3}$ & 3,01 & 1,17 \\
$\mathrm{Na}_{2} \mathrm{O}$ & 0,03 & 0,17 \\
$\mathrm{~K}_{2} \mathrm{O}$ & 0,85 & 0,79 \\
$\mathrm{CO}$ & 1,81 & 4,87 \\
$\mathrm{RI}$ & 0,26 & 0,79 \\
$\mathrm{CaO}$ & 1,63 & 1,19 \\
\hline
\end{tabular}

Tabela III - Características físicas dos cimentos Portland. [Table III - Physical characteristics of Portland cement.]

\begin{tabular}{|c|c|c|c|c|c|}
\hline Cimento & $\begin{array}{c}\# 200 \\
\text { NBR } \\
11579 \\
\%\end{array}$ & $\begin{array}{c}\# 325 \\
\text { NBR } \\
9202 \\
\%\end{array}$ & $\begin{array}{c}\text { Blaine } \\
\text { NBR } \\
7224 \\
\left(\mathrm{~cm}^{2} / \mathrm{g}\right)\end{array}$ & $\begin{array}{c}\text { Tempo } \\
\text { de pega } \\
\text { (min) } \\
\text { Início } \\
\text { Fim }\end{array}$ & $\begin{array}{c}\text { Resistência à } \\
\text { Compressão aos } \\
28 \text { Dias (MPa) }\end{array}$ \\
\hline CP II E & 32 & 7,9 & 3766,8 & $\begin{array}{l}164,7 \\
246,3\end{array}$ & 39,8 \\
\hline $\begin{array}{l}\text { CP V ARI } \\
\text { Plus }\end{array}$ & 0,17 & 2,24 & 4679,79 & $\begin{array}{l}150,78 \\
226,25\end{array}$ & 56,16 \\
\hline
\end{tabular}

\section{Aditivo}

O aditivo superplastificante possui as seguintes propriedades fornecidas pelo fabricante:

Função principal: Superplastificante; base química: melamina; aspecto: líquido; densidade: $1,11 \mathrm{~g} / \mathrm{cm}^{3}( \pm 0,02)$; pH: $8,5 \pm 1$; cor: alaranjado.

\section{Polímero acrílico}

O látex acrílico utilizado apresenta-se na forma de emulsão, aniônica isento de plastificantes, formulado a partir de um copolímero de éster de ácido acrílico e estireno. Características fornecidas pelo fabricante:

Natureza: dispersão aquosa aniônica de um copolímero de butilacrilato e estireno.

Teor de sólidos: 49,0 a 51,0\%; Viscosidade Brookfield (RVT $415{ }^{\circ} \mathrm{C}$ ): 1000-2000 mPas; Densidade: 1,02 g/ $\mathrm{cm}^{3}$; pH: 4,5 a 6,5

Temperatura mínima de formação do filme: $20^{\circ} \mathrm{C}$

Tamanho médio de partículas: $0,1 \mu \mathrm{m}$

Propriedades do filme: Aspecto límpido e transparente

Estabilidade ao envelhecimento e luz: boa

\section{Porcelanato}

Foram obtidas as características do porcelanato através de procedimentos experimentais desenvolvidos no Centro de Caracterização e Desenvolvimento de Materiais, CCDM/ UFSCar, conforme as respectivas normas. A NBR 13818 (1997) descreve os métodos de ensaio para a obtenção das características físicas e químicas de placas cerâmicas, especificados nos anexos A a V. Foram selecionados apenas os ensaios que avaliam as características diretamente relacionadas com a aderência da argamassa.

Absorção de água (NBR 13818 - Anexo B): 0,2\%

Expansão térmica linear (NBR 13818 - anexo K): $\alpha(25$ a $\left.325{ }^{\circ} \mathrm{C}\right)=70,9 \times 10^{-7}$

Choque térmico (NBR 13818 - anexo L, ensaio com imersão): Ausência de falhas visíveis após 10 ciclos. 
Tabela IV - Teores de sílica ativa e polímero acrílico. [Table IV - The mixing proportions of silica fume and polymer.]

\begin{tabular}{ccc}
\hline $\begin{array}{c}\text { Teor de sílica ativa } \\
\text { \% da massa } \\
\text { do cimento }\end{array}$ & $\begin{array}{c}\text { Látex acrílico } \\
\text { \% da massa } \\
\text { do cimento }\end{array}$ & $\begin{array}{c}\text { Denominação } \\
\text { da argamassa }\end{array}$ \\
\hline 5 & 5 & A1 \\
& 10 & A2 \\
& 15 & A3 \\
& 20 & A4 \\
\hline 10 & 5 & A5 \\
& 10 & A6 \\
& 15 & A7 \\
& 20 & A8 \\
\hline
\end{tabular}

\section{Preparo das argamassas}

Foram preparados oito traços de argamassas, conforme descritos na Tabela IV. Os materiais foram pesados e misturados em argamassadeira planetária. A proporção cimento:areia foi fixada igual 1:1,5 em massa, assim como a adição de superplastificante (1\%). A fim de garantir a plasticidade para a aplicação das argamassas, a água adicionada à mistura apresentou uma pequena variação. A água total, isto é, água adicionada mais água proveniente do látex foi mantida constante, obtendo-se a relação água total/ cimento igual a 0,4 para todas as argamassas.

Os procedimentos experimentais seguiram as prescrições constantes na NBR 14084 - Argamassa colante industrializada para assentamento de cerâmica - Determinação da resistência de aderência. O substrato padrão foi moldado conforme os procedimentos descritos na NBR 14082. Os moldes metálicos foram empregados para a obtenção de dimensões exatas e acabamento regular da superfície do substrato. As telas foram cortadas e fixadas no interior dos moldes utilizando-se espaçadores de plástico, que também garantiram o recobrimento da tela.

O conjunto substrato padrão, argamassa e porcelanato foi mantido sob cura ao ar em ambiente climatizado conforme norma até 28 dias. A determinação da resistência de aderência foi realizada aos 28 dias de aplicação da argamassa. Aos 27 dias, as pastilhas metálicas para a conexão com o equipamento de tração foram coladas com adesivo epóxi sobre as pastilhas de porcelanato. Após o endurecimento da cola entre a placa metálica e o porcelanato $(24 \mathrm{~h})$, foi realizado o ensaio de arrancamento por tração, seguindo os procedimentos descritos na NBR 14084.

\section{Determinação do diâmetro médio dos poros}

Foi determinado o diâmetro médio dos poros das argamassas A2, A4, A6 A8 especificadas na Tabela IV, a fim de avaliar o efeito das adições nesta propriedade e correlacionar esses valores com a resistência de aderência. Foram elaboradas pastas com os teores de adições das respectivas argamassas: $5 \%$ sílica ativa e 5,4\% de sólidos de polímero (A2); $5 \%$ de sílica ativa e 10,8\% de sólidos de polímero (A4); 10\% de sílica ativa e 5,4\% sólidos de polímero (A6); $10 \%$ de sílica ativa e 10,8\% de sólidos de polímero (A8). Foram moldados cilindros de diâmetro $5 \mathrm{~cm}$ e altura $10 \mathrm{~cm}$, curados sob as condições especificadas na norma NBR 14084, e após 26 dias foram cortados com serra diamantada nas dimensões do porta-amostra para a realização do ensaio de porosimetria por intrusão de mercúrio. Após o corte nas dimensões apropriadas, as amostras passaram por um processo de limpeza em ultrassom com imersão em álcool isopropílico, e secagem em estufa sob $50{ }^{\circ} \mathrm{C}$ por $24 \mathrm{~h}$. A tensão superficial do mercúrio foi igual a $0,485 \mathrm{~N} / \mathrm{mm}^{2}$, densidade do mercúrio $13,54 \mathrm{~g} / \mathrm{cm}^{3}$ e ângulo de contato entre o mercúrio e o sólido igual a $130{ }^{\circ} \mathrm{C}$.

\section{Obtenção de imagem por microscopia eletrônica de varredura}

As amostras das argamassas foram fraturadas para análise em microscópio eletrônico de varredura (MEV). As micrografias foram obtidas por detecção de elétrons secundários (baixa energia), pois são capazes de mostrar a morfologia da superfície fraturada.

\section{RESULTADOS E DISCUSSÃO}

Foram obtidos os valores da força de tração aplicada pelo equipamento para o arrancamento do porcelanato aplicado sobre as argamassas. Essa força é dividida pela área da pastilha e assim obtêm-se os valores da resistência de aderência (MPa). A Fig. 1 mostra os valores obtidos para as argamassas em estudo, os quais evidenciam que as adições de polímero e sílica ativa melhoram a resistência de aderência, aumentando com a quantidade de polímero e sílica-ativa.

Os valores do diâmetro médio dos poros das argamassas obtidos por porosimetria por intrusão de mercúrio são demonstrados na Fig. 2.

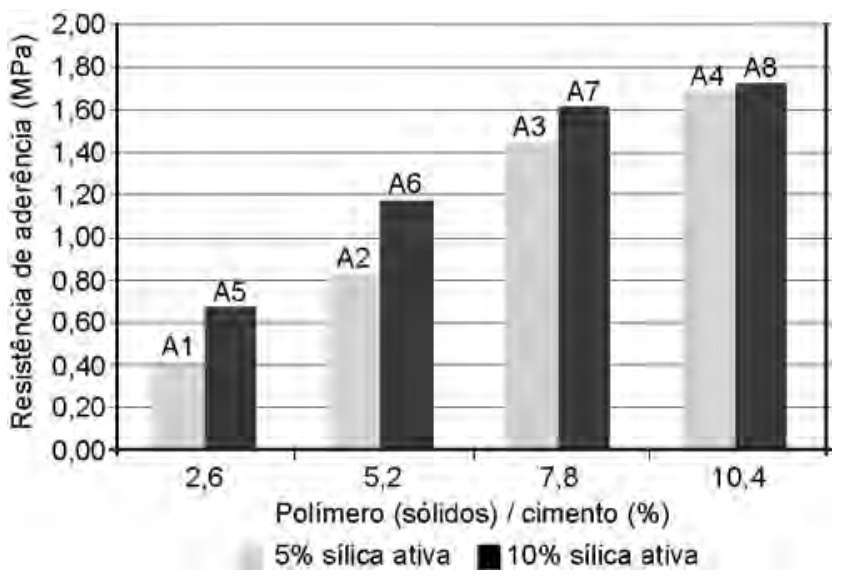

Figura 1: Resistência de aderência das argamassas em estudo. [Figure 1: Tensile adhesion strength of mortars.] 


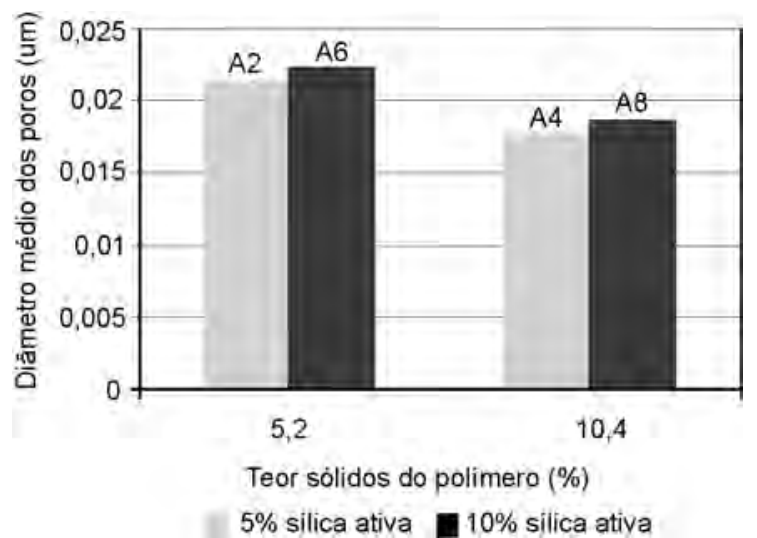

Figura 2: Diâmetro médio dos poros das argamassas A2, A4, A6 e A8. [Figure 2: Pore mean diameter of mortars A2, A4, A6 e A8.]

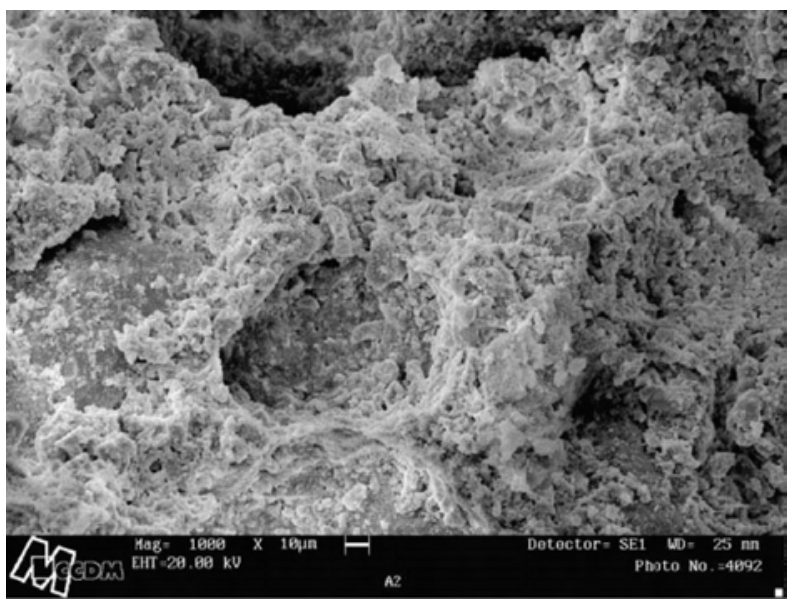

Figura 3: Imagem obtida por microscopia eletrônica de varredura da amostra fraturada de argamassa com 5\% de sílica ativa e 5,2\% de polímero (A2).

[Figure 3: Scanning electron microscopy of fractured specimens of mortars with $5 \%$ silica fume and $5.2 \%$ polymer (A2).]

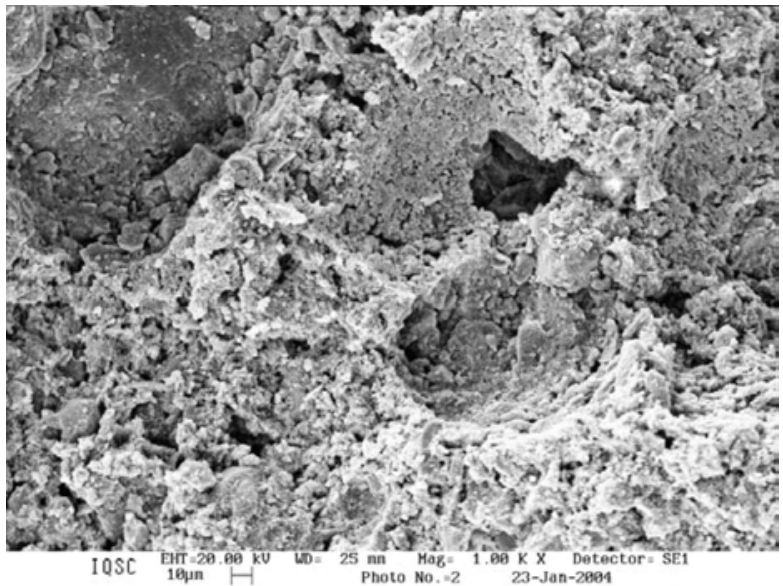

Figura 4: Imagem obtida por microscopia eletrônica de varredura da amostra fraturada de argamassa com 10\% de sílica ativa e 5,2\% de polímero (A6).

[Figure 4: Scanning electron microscopy of fractured specimens of mortars with $10 \%$ silica fume and 5.2\% polymer (A6).]

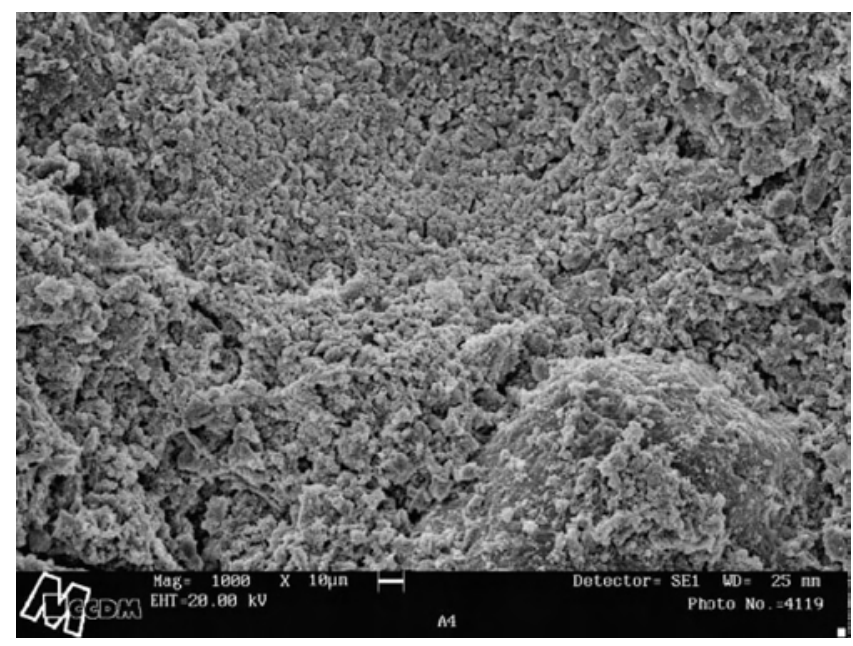

Figura 5: Imagem obtida por microscopia eletrônica de varredura de amostra fraturada da argamassa com $5 \%$ de sílica ativa e 10,4\% de polímero (A4).

[Figure 5: Scanning electron microscopy of fractured specimens of mortars with $5 \%$ silica fume and $10.4 \%$ polymer (A4).]

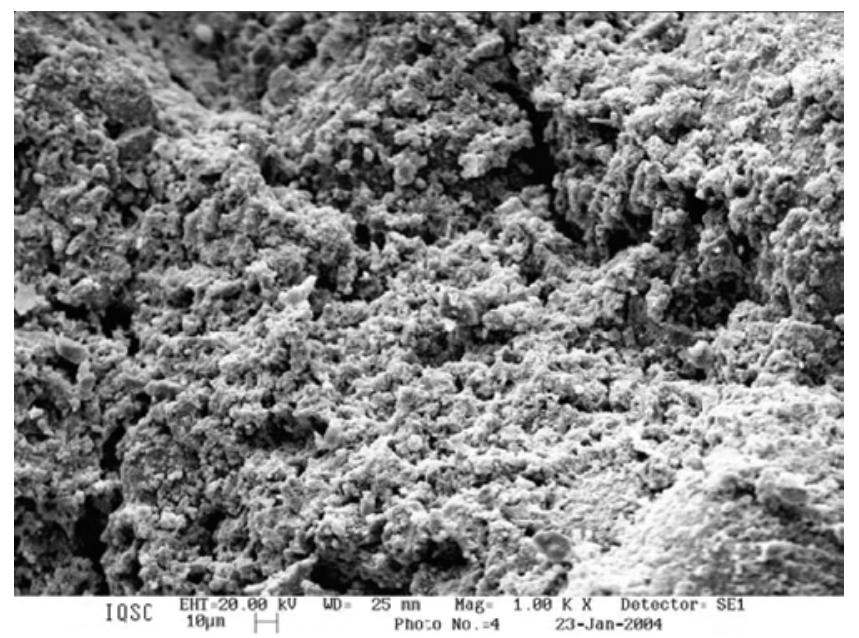

Figura 6: Imagem obtida por microscopia eletrônica de varredura de amostra fraturada da argamassa com 10\% de sílica ativa e 10,4\% de polímero (A8).

[Figure 6: Scanning electron microscopy of fractured specimens of mortars with $10 \%$ silica fume and $10.4 \%$ polymer (A8).]

Pode-se observar que as argamassas A2 e A6 apresentam o diâmetro médio dos poros bem próximos pois são constituídas pelo mesmo teor de polímero (5,2\%), assim como as argamassas A4 e A8, pois possuem o mesmo teor de polímero (10,4\%). Com base nos resultados é evidente que a adição de polímero causa uma diminuição no diâmetro médio dos poros.

As micrografias obtidas por microscopia eletrônica de varredura são observadas nas Figs. 3 a 6, conforme os teores de adição. As Figs. 3 e 4 (amostras A2 e A6, respectivamente) apresentam uma microestrutura irregular, com presença de vazios de grandes dimensões, quando comparados com os vazios apresentados nas imagens das Figs. 5 e 6 (amostras 
A4 e A8, respectivamente), as quais apresentam a morfologia dos compostos hidratados mais homogênea e regular.

\section{CONCLUSÕES}

O aumento da resistência de aderência é resultante da adição de polímero e sílica ativa às argamassas, conforme a Fig. 1, sendo que o aumento da aderência está relacionado com o aumento do teor de polímero e sílica ativa. Para altos teores de polímero (10,4\%), existe uma tendência de diminuir a influência da adição de sílica ativa.

A Fig. 2 demonstra que o incremento da aderência pode estar relacionado com o refinamento da estrutura dos poros das argamassas, ou seja, as adições promoveram a diminuição do diâmetro médio dos poros. As imagens obtidas por microscopia eletrônica de varredura evidenciam que a adição de polímero proporciona a microestrutura mais densa e homogênea, com vazios de menores dimensões, corroborando com os resultados obtidos por porosimetria. As Figs. 5 e 6 apresentam a microestrutura mais compacta e homogênea. As Figs. 3 e 4 caracterizam a microestrutura com a existência de vazios de grandes dimensões que diminuem a resistência da argamassa, além de diminuir a área de contato com o revestimento cerâmico.

\section{AGRADECIMENTOS}

À FAPESP pelo suporte financeiro.

\section{REFERÊNCIAS}

[1] C. Heck, Cerâmica Industrial 5 (1996) 21.

[2] A. P. N. Oliveira, Cerâmica Industrial 3 (1998) 34.

[3] J. Lavelle, ACI Mater. J. 85 (1988) 41.

[4] D. G. Walters, Concrete International: Design \& Construction 9 (1987) 44.

[5] Y. Ohama, Cement and Concrete Composites 20 (1998) 189.

[6] R. Ollitrault-Fichet, C. Gautier, G. Clamen, P. Boch, Cement and Concrete Res. 28 (1998) 1687.

[7] S. Chandra, P. Flodin, Cement and Concrete Res. 17 (1987) 875.

[8] P. Aïtcin, Concreto de Alto Desempenho, PINI, S. Paulo, Brasil (2000) 161.

[9] A. K. Chakraborty, S. C. Dutta, P. Sen, I. Ray, J. Polymer Mater. 17 (2000) 53.

[10] J. Gao, J. M. Qian, B. Wang, K. Morino, Cement and Concrete Res. 32 (2002) 41.

(Rec. 06/01/2006, Ac. 17/02/2006) 